\title{
The prevalence of Temporomandibular Disorder (TMD) and its severity among YARSI University Employees
}

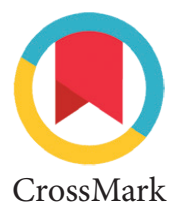

Helwiah Umniyati*

\section{Abstract}

Objective: This study described the prevalence of Temporomandibular Disorder (TMD) and its severity among YARSI University employees. Material and Methods: It was a cross sectional analytical study based on Fonseca's Questionnaire.

Results: The sample consisted of 225 participants, 118 of them were men (52.4\%) and 107 of them were women (47.6\%), with an average age of $37.99 \pm 12.05$ years old. Almost all of the samples (92\%) informed that they did not have a difficulty in mouth opening and moving the jaw to the sides (92\%), 84\% reported no clenching teeth and $80 \%$ reported no articulation disorders. While $34.8 \%$ of respondents reported muscular pain during mastication and $38.7 \%$ reported suffering from frequent headaches occasionally. Temporomandibular Joints (TMJ) clicking was reported among 13.3\% of participants and only a very small number of samples (4.9\%) reported pain in the temporomandibular joint.

Conclusion: The prevalence of TMD was quite high i.e $50.7 \%$. In term of severity, most of subjects had mild TMD's (86.8\%). The sociodemographic factors that significantly associated with severity of TMD were education and work status.
Department of Preventive and Public Health Dentistry, Faculty of Dentistry, University of YARSI, Central Jakarta, Indonesia
*Correspondence to: Helwiah Umniyati, Department of Preventive and Public Health Dentistry, Faculty of Dentistry, University of YARSI, Central Jakarta, Indonesia

helwiah.umniyati@yarsi.ac.id

Received: 1 July 2020

Revised: 2 July 2020 Accepted:

19 July 2020 Available Online:

1 August 2020

Keywords: Fonseca's questionnaire, Prevalence and severity, TMD

Cite this Article: Umniyati H. 2020. The prevalence of Temporomandibular Disorder Joint (TMD) and its severity among YARSI University Employees. Journal of Dentomaxillofacial Science 5(2): 82-85. D0I: 10.15562/jdmfs.v5i2.1074

\section{Introduction}

Temporomandibular joint disorders (TMD) describe as a number of related disorders that affect the temporomandibular joints (TMJ) and its associated muscles. ligaments, as well as dental occlusion. ${ }^{1}$ The prevalence of TMD varied from 9.8 to 80 percent and these problems are very common amongst age of 20-50 years, especially in women. ${ }^{2}$ In Brazilian study, Goncalves et $\mathrm{al}^{3}$ found that the prevalence of TMD was $39.2 \% .^{3}$ More than half of population in the world (60-70\%) have at least one of the symptoms of TMD in their lives, but very few of them seek treatment which is about 5\%. ${ }^{4}$ Signs and symptoms of TMJ disorders are pain in the jaw, difficulty swallowing or discomfort when swallowing, clicking or crepitation of the TMJs, limited jaw opening capacity and deviation in the movement patterns of the mandible, clicking or crepitation of the TMJs, jaw locked, headaches, uncomfortable bites, premature contact, and masticatory disorders. ${ }^{5,6}$ The etiology of TMD's involves several components which may work independently or together. These factors include genetics, stress, tooth occlusion changes, muscular dysfunction and systemic conditions that may manifest in the temporomandibular joint. ${ }^{6}$ TMD are more prevalence in age 20-45 years, with more females suffering TMD's than males. ${ }^{7}$ To measure the severity of
TMD, several instruments are used, among which the most widely used is the Fonseca anamnestic questionnaire and the Diagnostic Criteria for Temporomandibular Disorders (DC-TMD) assessment instruments. ${ }^{8}$ The purpose of our survey was to obtain the information on prevalence and severity of temporomandibular disorders among YARSI University employees in Jakarta, Indonesia, using the Fonseca's questionnaire.

\section{Material and Methods}

Using cross sectional analytic design we conducted a survey about TMD in YARSI University. Sampling was completed using the purposive method and the study sample consisted of 225 employees, including both administrative and teaching staff. The study sample had to meet the inclusion criteria, namely that respondents had never had prosthodontic treatment before. Exclusion criteria included respondents who use fixed orthodontics and those who had experienced TMJ trauma. The prevalence and severity of temporomandibular disorders were assessed using the Fonseca anamnestic index. ${ }^{8,9}$ The Fonseca Index is often used to look at the severity of TMD in individuals based on the following classifications: mild, moderate, severe and no TMD. The Fonseca questionnaire consists of 10 questions 
using a three point scale format (no, sometimes and yes). This questionnaire will check if there is pain in the temporomandibular joint, head \& back, while chewing, parafunctional habits, limited jaw movements, clicking, and perceptions of malocclusion \& emotional stress sensations. ${ }^{10}$ From all 10 questions, for each "yes" answer will be added and then multiplied by 10 , and the same way, for the answer "sometimes" will be added then multiplied by 5 , while for: "no" answer will be multiplied by 0 . After that all the scores is added to get the total score. The scores obtained are grouped into four categories, namely a $0-15$ score indicating there is no TMD, a score of 20-40 has a mild TMD, a score of 45-65 has a moderate TMD, and a score of 70100 has a severe TMD. ${ }^{10,11}$ Ethical approval was obtained from the Institutional Ethics Committee at YARSI University and the subjects were asked to sign informed consent before their participation in this study. Data analysis was carried out using the SPSS version 23. Pearson chi square test was used to assess the level of significance (critical p-value $<0.05)$.

\section{Results}

\section{Characteristics of Respondents}

From a total of 225 participants, males comprised $52.4 \%$ of the sample. The age of the study subjects varied from 18 years old to 72 years old, with the mean age of the respondents being 37.92 \pm 12.03 (SD) years old. Most of subjects had high education (61.3\%), worked as administrative staffs (74.7\%) table 1.

\section{Fonseca's Anamnestic Index Questions}

The distribution of participants according to their answers to the Fonseca Anamnestic Index, is shown in Table 2. There was a high prevalence of "No" answers to Index questions, which may be because the sample consists of a standard population.

Table 1 Frequency Distribution of Socio-demographic Factors

\begin{tabular}{llc}
\hline Socio-demographic & Number (\%) \\
\hline Gender & Males & $118(52.4 \%)$ \\
\multirow{2}{*}{ Age } & Females & $107(47.6 \%)$ \\
& $<30$ years & $68(30.2 \%)$ \\
& $30-40$ years & $67(29.8 \%)$ \\
Education & $>40$ years & $90(40.0 \%)$ \\
& Low ( $\leq$ Junior high school) & $87(38.7 \%)$ \\
Work Status & High (University) & $138(61.3 \%)$ \\
& Administrative staff & $168(74.7 \%)$ \\
& Faculty staffs & $57(25.3 \%)$ \\
\hline
\end{tabular}

The majority of the respondents reported that they did not had a difficulty in mouth opening and moving the jaw to the sides (92\%), while $34.8 \%$ of participants reported muscular pain during mastication and $38.7 \%$ of participants mentioned suffering from frequent headaches occasionally, and almost half of the respondents (43.6\%) reported no pain on neck. About $84 \%$ of respondents reported had no habits of clenching or grinding teeth and $80 \%$ of respondents reported no problems of tooth articulation. There were $13.3 \%$ of respondents reported clicking TMJ when opening their mouths and chewing food. Few of the samples $(4.9 \%)$ reported pain in the temporomandibular joint and about $10.2 \%$ of the sample felt themselves tense and $28.9 \%$ as occasionally tense/nervous person table 2 .

From this study we found the most common symptoms for TMD were TMJ sound while chewing/opening mouth (46.2\%), fatigue/muscle pain during mastication $(34.8 \%)$ and pain in TMJ (25.3\%). Other signs that were not directly related to TMD were neck pain (56.4\%), headaches (52.4\%), and emotional stress (39.1\%).

\section{TMD's Prevalence and Its Severity}

According to the Fonseca grading, out of 225 participants, 49.3\% reported no TMD's and $50.7 \%$ reported TMD's. Please refer to figure 1 for more details.

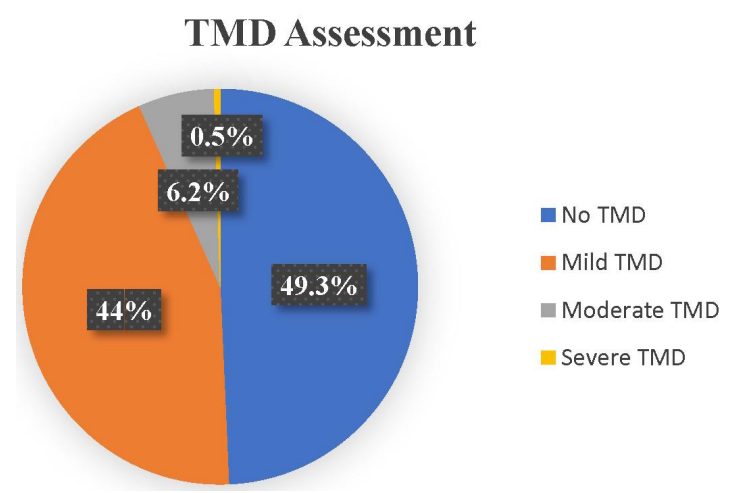

Figure 1 TMD Assessment

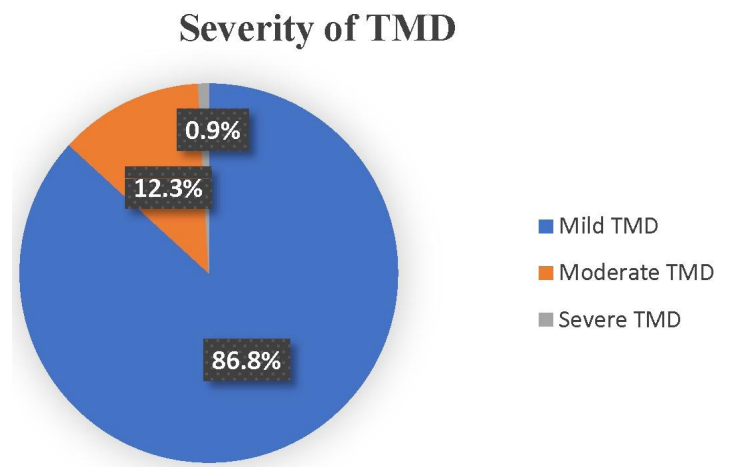

Figure 2 Severity of TMD 
Table 2 Distribution Answers of Fonseca's Anamnestic Index Questions

\begin{tabular}{lccc}
\hline Fonseca's Anamnestic Index & No & Sometimes & Yes \\
\hline Difficulty opening mouth wide & $207(92.0 \%)$ & $16(7.1 \%)$ & $2(0.9 \%)$ \\
Difficulty moving jaw to the sides & $207(92.0 \%)$ & $16(7.1 \%)$ & $2(0.9 \%)$ \\
Fatigue /Pain during mastication & $148(65.2 \%)$ & $69(31.2 \%)$ & $8(3.6 \%)$ \\
Headaches & $107(47.6 \%)$ & $87(38.7 \%)$ & $31(13.8 \%)$ \\
Neck pain & $98(43.6 \%)$ & $96(42.7 \%)$ & $31(13.8 \%)$ \\
TMJ pain & $168(74.7 \%)$ & $46(20.4 \%)$ & $11(4.9 \%)$ \\
TMJ sound (clicking) & $121(53.8 \%)$ & $74(32.9 \%)$ & $30(13.3 \%)$ \\
Problem of tooth articulation & $180(80.0 \%)$ & $9(4.0 \%)$ & $36(16.0 \%)$ \\
Habits of clenching or grinding teeth & $189(84.0 \%)$ & $17(7.6 \%)$ & $19(8.4 \%)$ \\
Perception as a nervous person & $137(60.9 \%)$ & $65(28.9 \%)$ & $23(10.2 \%)$
\end{tabular}

Table 3 Association between severity and sociodemographic factors

\begin{tabular}{|c|c|c|c|c|c|}
\hline Socio-demographic & No TMD & Mild & Moderate & Severe & P-Value \\
\hline \multicolumn{6}{|l|}{ Gender } \\
\hline Males & $51(43.2 \%)$ & $59(50.0 \%)$ & $7(5.9 \%)$ & $1(0.8 \%)$ & \multirow{2}{*}{0.183} \\
\hline Females & $60(56.1 \%)$ & $40(37.4 \%)$ & $7(6.5 \%)$ & $0(0 \%)$ & \\
\hline \multicolumn{6}{|l|}{ Age } \\
\hline$<30$ years & $30(44.1 \%)$ & $32(47.1 \%)$ & $5(7.4 \%)$ & $1(1.5 \%)$ & \multirow{3}{*}{0.683} \\
\hline $30-40$ years & $32(47.8 \%)$ & $31(46.3 \%)$ & $4(6.0 \%)$ & $0(0.0 \%)$ & \\
\hline$>40$ years & $49(54.4 \%)$ & $36(40.0 \%)$ & $5(5.6 \%)$ & $0(0.0 \%)$ & \\
\hline \multicolumn{6}{|l|}{ Education } \\
\hline Low & $31(35.6 \%)$ & $45(51.7 \%)$ & $10(11.5 \%)$ & $1(1.1 \%)$ & \multirow{2}{*}{0.002} \\
\hline High & $80(58.0 \%)$ & $54(39.1 \%)$ & $4(2.9 \%)$ & $0(0.0 \%)$ & \\
\hline \multicolumn{6}{|l|}{ Work Status } \\
\hline Administrative staff & $68(40.5 \%)$ & $85(50.6 \%)$ & $14(8.3 \%)$ & $1(0.6 \%)$ & \multirow{2}{*}{0.000} \\
\hline Faculty staffs & $43(75.4 \%)$ & $14(24.6 \%)$ & $0(0 \%)$ & $0(0 \%)$ & \\
\hline
\end{tabular}

When we looked at subjects who had TMD, we divided the TMD according to severity, most of the participant had mild TMD $(86.8 \%)$ as seen in figure 2 .

In cross tabulation between gender and TMD, there was no significant difference between males and females although more males presented with TMD. Based on age, it looks like the younger participants had a higher prevalence of TMD, but the relationship was not significant. There was a significant relationship between education and TMD, lower educated respondents had presented with more TMD than those with higher education $(\mathrm{p}=0.002)$. According to the work status, the administrative staff had a higher prevalence of TMD than faculty staff and the relationship was determined to be significant (p-value $<0.000$ ). For more details, refer to table 3.

\section{Discussion}

This study revealed that $50.7 \%$ of respondents had TMD's, with half of respondents having some sign and symptom of the disorder. Using the same Fonseca's questionnaire, many studies also found a similar prevalence. Nomura et al. study ${ }^{6}$ was conducted on 218 dental students from Brazil found that $53.2 \%$ of the students experienced TMD. Pedroni et al. ${ }^{12}$ obtained an even higher prevalence by using the same questionnaire as they found a prevalence of $68 \%$ Brazilian University students.

In this study, we found that the most common symptoms of TMD were noise in the TMJ while chewing/opening mouth, fatigue/muscle pain when chewing and pain in the TMJ. These results are in line with the findings of Karthik et al. ${ }^{8}$ who used the same questionnaire. Karthik et al. ${ }^{8}$ results included pain while chewing, TMJ pain and TMJ clicking while chewing/opening mouth. ${ }^{8}$

We also found that other dominant signs that were not directly related to TMD which were neck pain, headaches, and participant's perception as a nervous/tense person. Arsalan Wahid et al. found similar results. They also identified tenseness, 
headache and stiff neck ${ }^{11}$ as factors, and these were in line with the results of Nomura et al. ${ }^{6}$ Kassab et al. ${ }^{13}$ found that TMJ sounds, TMJ pain, and pain in mandibular movements were the most frequently reported signs and symptoms. ${ }^{13}$ Campos et al. ${ }^{15}$ revealed that the most common signs and symptoms reported were headache, neck pain/stiff neck, habits clenching/grinding teeth, problem of tooth articulation, feel fatigue/muscle pain during chewing and tense/nervous. ${ }^{10}$ Bevilaqua-Grossi et al. ${ }^{14}$ emphasized that pain during mastication, TMJ pain, and TMJ sounds were good predictors of TMD, while neck pain and headache proved to be poor indicators to distinguish TMD severity. ${ }^{14}$

Our study revealed that males had a higher prevalence than females, although there was no statistical significance in sex differences. This finding was among dental students of Khyber College in Pakistan. ${ }^{9}$ Other research supports that females were more affected than males, including the research of Nomura et al. ${ }^{6}$ Karthik et al. ${ }^{8}$ and Bevilaqua-Grossi et al. ${ }^{14}$ For the age category, no significant association between age and TMDs was found. This result was also support by Karthik et al. ${ }^{8}$ It appears that the younger aged participants had a higher prevalence of TMD. This finding was in line with the statement that TMD occurs predominantly in young adults. ${ }^{3,15}$ Education and work status in this study were also in line with the results of other research. Higher educated respondents (faculty staff) had a lower prevalence of TMD than lower educated respondents (administrative staff). ${ }^{16}$

\section{Conclusion}

From this study the prevalence of TMD was quite high i.e $50.7 \%$. In term of severity, most of them had mild TMD's (86.8\%). The sociodemographic factors that significantly associated with severity of TMD were education and work status.

\section{Acknowledgment}

The author would like to thank all of YARSI's employees for their participation in this study and also a great thanks to my students: Riska Juniar, Sinta Primanita Amanah, Rahma Putri Kartikasari and Vannia Vegafriasya who helped in the collection of the data.

\section{Conflict of Interest}

The authors report no conflict of interest.

\section{References}

1. Bonjardim LR, Lopes-Filho RJ, Amado G, et al. Association between symptoms of temporomandibular disorders and gender, morphological occlusion and psychological factors in a group of university students. Indian J Dent Res 2009;20: 190-194.

2. Feteih RM. Signs and symptoms of temporomandibular disorders and oral parafunctions in urban Saudi arabian adolescents: a research report. Head \& Face Medicine 2006;2: 25.

3. Goncalves DA, Dal Fabbro AL, Campos JA, et al. Symptoms of temporomandibular disorders in the population: an epidemiological study. J Orofac Pain 2010;24: 270-278.

4. Macfarlane TV, Gray RJ, Kincey J, et al. Factors associated with the temporomandibular disorder, Pain Dysfunction Syndrome (PDS): Manchester case-control study. Oral Dis 2001;7: 321-330.

5. Murphy MK, MacBarb RF, Wong ME, et al. Temporomandibular joint disorders: a review of etiology, clinical management, and tissue engineering strategies. The Int J Oral Maxillofac Implants 2013;28: 393-414.

6. Nomura K, Vitti M, Oliveira AS, et al. Use of the Fonseca's questionnaire to assess the prevalence and severity of temporomandibular disorders in Brazilian dental undergraduates. Braz Dent J 2007;18: 163-167.

7. Roda R, Bagán J, Fernández J, et al. Review of temporomandibular joint pathology. Part I: classification, epidemiology and risk factors. Med Oral Patol Oral Cir Bucal 2007;12: 292-298.

8. Karthik R, Hafila MIF, Saravanan C, et al. Assessing prevalence of temporomandibular disorders among university students: a questionnaire study. JISPCD 2017;7: 24-29.

9. Niazi QFA, KHAN M, Rehman AU. Frequency and severity of temporomandibular disorders amongst dental students of Khyber College of Dentistry. Pak Oral Dental J 2017;37: 403-406.

10. Campos JADB, Carrascosa AC, Bonafé SFS, et al. Severity of temporomandibular disorders in women: validity and reliability of the Fonseca Anamnestic Index. Braz Oral Res (Sao Paulo) 2014;28: 16-21.

11. Wahid A, Mian FI, Razzaq A, et al. Prevalence and Severity of Temporomandibular Disorders (TMD) in Undergraduate Medical Students using Fonseca's Questionnaire. Pak Oral Dental J 2014;34: 1.

12. Pedroni CR, De Oliveira AS, Guaratini MI. Prevalence study of signs and symptoms of temporomandibular disorders in university students. J Oral Rehabil 2003;30: 283-289.

13. Kassab M, Bakry A, Salim WS. The incidence of temporomandibular joint disorders among dental students in Aljouf University, KSA. IIJMMS 2015;2: 5-11.

14. Bevilaqua-Grossi D, Chaves TC, de Oliveira AS, et al. Anamnestic index severity and signs and symptoms of TMD. Cranio 2006;24: 2.

15. Campos JADB, Carrascosa AC, Bonafé FSS, et al. Epidemiology of severity of temporomandibular disorders in Brazilian women. J Oral Facial Pain H 2014;28: 147-152.

16. Mude AH, Irsal I. Management of myofascial pain: a systematic review. J Dentomaxillofac Sci 2020;5: 1-7.

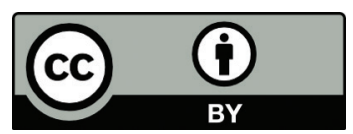

This work is licensed under a Creative Commons Attribution 